\title{
Smeared antibranes polarise in AdS
}

\section{Fridrik Freyr Gautason, Brecht Truijen and Thomas Van Riet}

Instituut voor Theoretische Fysica, K.U. Leuven, Celestijnenlaan 200D B-3001 Leuven, Belgium

E-mail: ffg@fys.kuleuven.be, brecht.truijen@fys.kuleuven.be, thomas.vanriet@fys.kuleuven.be

ABSTRACT: In the recent literature it has been questioned whether the local backreaction of antibranes in flux throats can induce a perturbative brane-flux decay. Most evidence for this can be gathered for $\mathrm{D} 6$ branes and $\mathrm{D} p$ branes smeared over $6-p$ compact directions, in line with the absence of finite temperature solutions for these cases. The solutions in the literature have flat worldvolume geometries and non-compact transversal spaces. In this paper we consider what happens when the worldvolume is AdS and the transversal space is compact. We show that in these circumstances brane polarisation smoothens out the flux singularity, which is an indication that brane-flux decay is prevented. This is consistent with the fact that the cosmological constant would be less negative after braneflux decay. Our results extend recent results on $\mathrm{AdS}_{7}$ solutions from D6 branes to $\mathrm{AdS}_{p+1}$ solutions from $\mathrm{D} p$ branes. We show that supersymmetry of the AdS solutions depend on $p$ non-trivially.

KEYwords: Flux compactifications, dS vacua in string theory, D-branes

ARXiv EPrint: 1502.00927 


\section{Contents}

1 Introduction 1

2 Anti-D3 solutions 3

2.1 Ansatz 3

2.2 Fully smeared 3-branes 4

2.3 Compact AdS solutions 5

2.4 Non-compact flat solutions $\quad 6$

3 Spherical 5-branes $\quad 7$

3.1 D5 polarisation 8

$\begin{array}{lll}3.2 & \text { NS5 polarisation and flux decay } & 9\end{array}$

4 Anti-D $p$ solutions $\quad 11$

4.1 The AdS curvature 12

$\begin{array}{lll}4.2 & \text { Flat solutions } & 13\end{array}$

$\begin{array}{lll}4.3 & \text { Brane polarisation } & 13\end{array}$

$\begin{array}{llr}5 & \text { Conclusion } & 16\end{array}$

$\begin{array}{ll}\text { A Notation and conventions } & 17\end{array}$

$\begin{array}{lr}\text { B Second-order equations } & 18\end{array}$

$\begin{array}{ll}\text { C Supersymmetric AdS } & 19\end{array}$

\section{Introduction}

In recent years there has been an active study of supergravity solutions that feature $\mathrm{D} p$ branes locally surrounded by fluxes that induce a delocalised $\mathrm{D} p$ charge density of the opposite sign to the brane charge. These different signs can be seen in the Bianchi identity:

$$
\mathrm{d} F_{8-p}=H \wedge F_{6-p}+Q \delta_{8-p} .
$$

The solutions of interest are such that the orientation of the first form on the r.h.s. of (1.1) is opposite to second one. For this reason we name those branes "antibranes" where the "anti" refers to the charge being opposite to the charge density in the fluxes.

Solutions with this distinctive property can be categorised into two classes: 1) nonsupersymmetric solutions with flat $\mathrm{D} p$ worldvolume and 2) solutions with a $\mathrm{D} p$ worldvolume that is AdS and a transversal space that is potentially compact. The AdS solutions can be supersymmetric [1], but do not need to be [2] 
Examples of the first kind are the non-compact geometries in which supersymmetry (SUSY) is broken by the brane, with the prime example being anti-D3 branes in the Klebanov-Strassler throat [3-5] first studied in [6]. When combined with orientifolds and quantum corrections there is a belief that it can be made into a compactification geometry for which the SUSY-breaking branes are used to uplift AdS vacua to dS vacua [7] or as a base for brane inflation [8].

Examples of the second kind are warped $\mathrm{AdS}_{7}$ vacua in massive IIA SUGRA built from spacetime-filling D6 branes and a transversal space that is conformal to an $S^{3}$. These solutions were first uncovered in $[9,10]$, but their supersymmetry together with more details was properly understood in [1]. Furthermore, these solutions provide a concrete gravity dual to six-dimensional $(1,0)$ SCFT's [11].

Both classes of solutions feature a peculiar property that has been the origin of an ongoing debate: due to the differences in charges, the fluxes are electromagnetically (and gravitationally) attracted to the branes in such a way that a singular flux cloud is formed around the branes [9, 12-14]. This was first uncovered in [3, 4] and by now a vast literature on this exists, with a formal proof for this unavoidable singularity presented in $[15,16] .{ }^{1}$ Since fluxes can materialise into actual branes [6], one is tempted to conclude that a singular, or large, flux pile-up leads to a quick annihilation of the flux with the antibrane, possibly making the solution perturbatively unstable $[13,19]$ (see also [14] for recent comments on this). This picture is strengthened by the absence of regular solutions at finite temperature with flat worldvolume [16, 20, 21].

Recently this interpretation of a perturbative decay has been challenged by some good arguments $[14,22]$. First of all it has been claimed that the singularity will get resolved by stringy corrections in such a way that the resulting flux clumping is small enough for at least a single antibrane to be meta-stable [14]. This can very well be correct and is currently under investigation, but it would clearly be more gratifying if the singularity can be resolved at supergravity length scales, such that the original arguments for antibrane meta-stability [6] are applicable. Interestingly a resolution at sufficiently large length scales has been argued by Hartnett in [22]. The basic claim of [22] is that the nogo-theorem for finite temperature resolutions of [16] can be circumvented. Secondly a simplified trick was found to understand the local geometry of localised antibranes and it seems to indicate that a Polchinski-Strassler (PS) type of singularity resolution [23] will take place that dilutes the flux clumping strongly enough to prevent direct brane-flux decay. This is in contrast with earlier investigations of a possible PS resolution that turned out not to work [24, 25], and according to [22] the reasons for this is the use of smeared antibranes ${ }^{2}$ instead of localised ones. It would be interesting to verify this explicitly. In this paper we do consider smeared antibranes but we demonstrate that, when they live in AdS space, their singularities do get resolved as opposed to flat space. In that sense there is no disagreement in the literature

\footnotetext{
${ }^{1}$ Around orientifolds this phenomenon does not occur since they repel the flux gravitationally as much as they attract it electromagnetically [17]. This nicely fits together with orientifold compactifications where orientifolds in fluxes of opposite charge are the basic ingredients [18].

${ }^{2}$ For the anti-D6 solutions [10] no smearing was used and the absence of a PS resolution is not questioned in this particular case.
} 
when it comes to flat space smeared anti-Dp branes or localised anti-D6 branes: the absence of regular finite $\mathrm{T}$ solutions seems without doubt and perfectly in line with the "no PSresolution' results. Hence perturbative brane-flux decay might very well be what is going on in the flat space solutions, but is inconsistent with the AdS solutions. Indeed, upon brane-flux decay these would have a less negative CC and hence more energy, so one expects those solutions to be stable.

In the case of the anti-D6 brane this has been understood by now. For the supersymmetric $\mathrm{AdS}_{7}$ solutions it was found that the (anti-)D6-branes polarise into spherical D8 branes $[1,2]$, which resolves the flux singularity since a charged sphere attracts the flux in a more delocalised fashion. This polarisation does not occur for the flat SUSY-breaking solutions [24] and we expect the latter solutions to decay perturbatively before the flux reaches the singular values. In this paper we extend this picture to the other branes and uncover that a similar story holds (up to certain subtleties): the compact $\operatorname{AdS}_{p+1}$ solutions are such that the $\mathrm{D} p$ branes polarise into spherical $\mathrm{D}(p+2)$ branes and brane-flux decay does not occur. For D3 branes brane-flux decay can be studied very explicitly since in that case it proceeds through brane polarisation into an NS5 brane [6] in a direction orthogonal to the D5 polarisation. For the other branes it is unclear what the explicit brane-flux decay mechanism is, most likely it is related to a T-dual version of spherical NS5-branes.

The rest of this paper is organised as follows. In section 2 we discuss smeared anti-D3 solutions and the corresponding $\mathrm{AdS}_{4}$ vacua and in section 3 we verify whether polarising into spherical D5 branes resolves the singularities and whether brane-flux decay can be prevented. In section 4 we generalise the discussion to $\mathrm{D} p$ branes smeared over (6 $p$ ) directions and we conclude in section 5 . We have added various appendices which include for example a discussion of the constraints that SUSY puts on the AdS solutions in this paper.

\section{Anti-D3 solutions}

In this section we describe the compact $\mathrm{AdS}_{4}$ solutions build from anti-D3 branes whose $\mathrm{RR}$ tadpole is canceled by 3-form fluxes and non-compact anti-D3 solutions with flat worldvolumes that do not require an $\mathrm{RR}$ tadpole cancellation. The existence of the $\mathrm{AdS}_{4}$ solutions found here were established in the limit of smeared antibranes in [26] (and generalised to other dimensions in [17]). We have not yet made an attempt to construct the solutions with fully localised branes but we expect them to be contained in the analysis of [27]. The reader interested in checking the calculations will find use of appendix A that fixes notation and conventions.

\subsection{Ansatz}

We consider the following Ansatz for the metric:

$$
\mathrm{d} s^{2}=\mathrm{e}^{2 A} \mathrm{~d} s_{\mathrm{AdS}_{4}}^{2}+\mathrm{e}^{2 B} \mathrm{~d} s_{S^{3}}^{2}+\mathrm{e}^{2 C}\left(\mathrm{~d} \rho^{2}+\mathrm{e}^{2 D} \mathrm{~d} \Omega^{2}\right),
$$

where $\mathrm{d} s_{\mathrm{AdS}_{4}}^{2}, \mathrm{~d} s_{S^{3}}^{2}$ and $\mathrm{d} \Omega^{2}$ are the metrics for $\mathrm{AdS}_{4}$, the three-sphere $S^{3}$ and two-sphere $S^{2}$ respectively. These metrics are chosen such that the corresponding unwarped Ricci 
tensors take the canonical form

$$
\hat{R}_{\mu \nu}=\Lambda \hat{g}_{\mu \nu}, \quad \hat{R}_{m n}=2 \hat{g}_{m n}, \quad \hat{R}_{i j}=\hat{g}_{i j} .
$$

Here the hatted variables denote the unwarped quantities:

$$
\mathrm{d} s_{\mathrm{AdS}_{4}}^{2}=\hat{g}_{\mu \nu} \mathrm{d} x^{\mu} \mathrm{d} x^{\nu}, \quad \mathrm{d} s_{S^{3}}^{2}=\hat{g}_{m n} \mathrm{~d} x^{m} \mathrm{~d} x^{n}, \quad \mathrm{~d} \Omega^{2}=\hat{g}_{i j} \mathrm{~d} x^{i} \mathrm{~d} x^{j},
$$

these equations also serve to fix the index conventions that we use throughout the paper. The warp factors $A, B, C$ and $D$ are only functions of the coordinate $\rho$ and so the Einstein equations reduce to ordinary differential equations for the warp factors. We parametrize our non-zero fluxes as follows:

$$
\begin{aligned}
F_{3} & =M \mathrm{vol}_{3}, \\
H & =-\lambda \mathrm{e}^{\phi} \star_{6} F_{3}, \\
F_{5} & =\left(1+\star_{10}\right) \star_{6} \mathrm{e}^{-4 A} \mathrm{~d} \alpha .
\end{aligned}
$$

Like the warp factors, $\lambda, \alpha$ and $\phi$ are functions of the $\rho$ coordinate, whereas $M$ is a constant topological flux quantum. The volume form $\mathrm{vol}_{3}$ is the volume form on the unit three-sphere. The equations of motion turn into a system of coupled ordinary differential equations (ODE's) and are written out explicitly in appendix B. From the equation of motion for $H$ it immediately follows that

$$
\alpha=\lambda \mathrm{e}^{4 A} .
$$

In the case where $\lambda$ is \pm 1 the 3 -form fluxes combine into ISD or anti-ISD flux as in [18] but this is not true in general due to the non-trivial 5 -form field strength. The Bianchi identity for $F_{5}$ takes the form

$$
\mathrm{d} F_{5}-H \wedge F_{3}=N_{\mathrm{D} 3} \mu_{3} \delta_{6},
$$

where $\delta_{6}$ is a 6 -form with delta function support on the worldvolume of the branes. Notice that we have added an integer $N_{\mathrm{D} 3}$ to allow for a stack of D3-branes. Integrating the Bianchi identity over the internal space, we obtain the tadpole cancellation condition,

$$
\int F_{3} \wedge H=Q
$$

where $Q$ denotes the total brane charge. It is clear that even though we have included the effect of D3-branes in the Bianchi identity (2.6), the form of the metric (2.1) does not allow for fully localised branes. Indeed the branes are smeared over the three-sphere, but are localised at $\rho=0$.

\section{$2.2 \quad$ Fully smeared 3-branes}

A fully smeared limit of the solution can be obtained by replacing the delta function in equation (2.6) with its integrated average

$$
\delta_{6} \rightarrow \operatorname{vol}_{6},
$$


where the internal volume form $\operatorname{vol}_{6}$ is unwarped and normalized to 1 . This solution has previously been described in [26] (see also [17]). The smearing has the effect of restoring symmetry in the internal manifold so it reduces to an exact product space of two spheres. This means that $\mathrm{e}^{2 D}=\sin ^{2} \rho$ and all other warp factors and functions are constant. Therefore we find that $F_{5}$ vanishes and the function $\lambda$ introduced in equation (2.4) takes a constant value $\lambda=1$. This corresponds precisely to the combined 3 -form flux

$$
G_{3}=F_{3}-i \mathrm{e}^{-\phi} H
$$

being imaginary self-dual, i.e. $\star_{6} G_{3}=-i G_{3}$. The size of the 3 -form flux is fixed by $(2.6)$ to be

$$
Q=g_{s}\left|F_{3}\right|^{2}
$$

The charge conjugated solution would have $Q=-g_{s}\left|F_{3}\right|^{2}$ and $\lambda=-1$ which corresponds to anti-ISD fluxes. The expression for the warped cosmological constant is

$$
\mathrm{e}^{-2 A} \Lambda=-\frac{1}{4} Q
$$

which shows that if the brane charge $Q$ would decrease, the total vacuum energy would increase. So already here we notice that a decay process which eliminates $Q$ against $M$ such that the tadpole cancelation condition (2.7) is still satisfied cannot occur.

\subsection{Compact AdS solutions}

The fully smeared solutions make it clear that compactness is only possible when the worldvolume of the brane is AdS. Flat solutions are necessarily non-compact. It is wellknown that for non-compact solutions there is no relation between the CC of the base space and the fluxes. In compact solutions the size of the $\mathrm{CC}$ is determined by the energy, that in turn is determined by the fluxes and the branes. In this subsection we look at compact AdS solutions.

We derive the relation between the CC and the fluxes using the results of [15]. This computation requires the gauge potential $C_{4}$. We can choose a gauge for $C_{4}$ for which the external part, $C_{4}^{\text {ext }}$, vanishes at the position of the branes, $\rho=0$,

$$
C_{4}=B \wedge C_{2}-\left(\alpha-\alpha_{0}\right) \operatorname{vol}_{4} .
$$

Recall that volume forms such as $\operatorname{vol}_{4}$ are always defined without warp factors. The equation for the cosmological constant of the external spacetime can be expressed as follows

$$
\Lambda=\frac{1}{4 V_{6}}\left[N_{\mathrm{D} 3} S_{\mathrm{loc}}+\frac{1}{V_{4}} \int H \wedge\left(\mathrm{e}^{-\phi} \star_{10} H+F_{3} \wedge C_{4}^{\mathrm{ext}}\right)\right],
$$

where $V_{4}$ and $V_{6}$ are "volumes" of the external and internal spaces, defined as follows

$$
V_{4}=\int \mathrm{vol}_{4}, \quad V_{6}=\int \star_{6} \mathrm{e}^{2 A} .
$$


The gauge choice for $C_{4}$ is such that, on-shell, the first term in the bracket drops out and we are left only with the flux integral in the second term. This reduces to

$$
\Lambda=\frac{\alpha_{0}}{4 V_{6}} \int H \wedge F_{3}=-\frac{Q \alpha_{0}}{4 V_{6}},
$$

where we made use of (2.7). Crucially this shows that the function $\alpha$ takes a nonvanishing value at the position of a D3-brane, this means that $\lambda$ which relates $F_{3}$ and $H$ in (2.4) has the asymptotic behaviour close to the brane:

$$
\lambda \underset{\rho \rightarrow 0}{\longrightarrow}-\frac{4 V_{6} \Lambda}{Q} \mathrm{e}^{-4 A} .
$$

This obviously blows up since the warp factor $\mathrm{e}^{2 A}$ vanishes in the vicinity of the brane. Combined with the fact that $F_{3}$ is constant 3 -form flux, this leads to the, by now well-known fact that the energy density of $H$ has a singular behaviour close to the brane.

In deriving (2.15) we have assumed that the solution is compact, although no proof for this exists, apart from the observation that the fully smeared solution is compact. To show that compact solutions can exist one should numerically evaluate the coupled ODE's from appendix $\mathrm{B}$, which we have not done and leave for future investigation. We verify in appendix $\mathrm{C}$, that unlike the $\mathrm{AdS}_{7}$ solution from anti-D6 branes, the compact $\mathrm{AdS}_{4}$ solution from partially smeared anti-D3 branes cannot be supersymmetric.

\subsection{Non-compact flat solutions}

Compactness enforces the solutions to be AdS but once we give up compactness, we can consider flat solutions. Flat solutions are interesting from the point of view of antibrane SUSY-breaking [6, 7, 28]. Antibrane SUSY-breaking, at least for anti-D3 branes, can be studied explicitly in the Klebanov-Strassler (KS) throat [29]. The KS throat is supersymmetric and regular at the tip and therefore it makes a perfect background to add the singular SUSY-breaking source at the tip. The full solution is out of reach and most likely will remain out of reach, but when the anti-D3 branes are smeared over the tip, the equations of motion, that describe the backreaction become ODE's and approximate solutions have been found $[4,30]$. Close to the tip the details of the singular flux clumping are nicely captured by a much simpler background [21], which is the T-dual to the flat antiD6 solution [10]. The only difference from our previous Ansatz are the curvature of the metric factors

$$
\mathrm{d} s^{2}=\mathrm{e}^{2 A} \mathrm{~d} s_{\text {Mink }_{4}}^{2}+\mathrm{e}^{2 B} \mathrm{~d} s_{\mathbb{T}^{3} / S^{3}}^{2}+\mathrm{e}^{2 C}\left(\mathrm{~d} \rho^{2}+\mathrm{e}^{2 D} \mathrm{~d} \Omega^{2}\right),
$$

where $\mathrm{d} s_{\text {Mink }_{4}}^{2}, \mathrm{~d} s_{\mathbb{T}^{3} / S^{3}}^{2}$ and $\mathrm{d} \Omega^{2}$ are the metrics for $\mathrm{Mink}_{4}$, the three-torus $\mathbb{T}^{3}$ or the 3 sphere $S^{3}$ and two-sphere $S^{2}$ respectively. The solutions with the torus factor can be obtained from T-duality of the anti-D6 solution. When it comes to the physics of flux clumping there is no real difference between the solutions with the torus factor and the solutions with the $S^{3}$ factor. The solutions with the $S^{3}$ factor are however more insightful since there is a more explicit picture for brane-flux annihilation in that case [6]. 
Also here it can easily be demonstrated that $\lambda$ blows up and hence there is singular flux clumping [21]. When it comes to the local physics associated to the flux clumping, this model captures exactly the same physics as the model with anti-D3's smeared over the tip of the $S^{3}$ in $\mathrm{KS}$; for instance it can be shown that the polarisation potential for spherical D5 branes in both cases is almost identical [25]. In the next section we compute the polarisation potential both for the polarisation into D5 and NS5 branes.

Due to the non-compactness, we can decouple the size of the $\mathrm{CC}$ from the brane charges and the flux quanta, we are free to chose a value for the CC. It is not necessary to take it to be exactly zero. The main result of this paper is that when the CC is set by the brane charges and fluxes, polarisation will occur. When the CC is parametrically different, which can be done for non-compactifications, or in KKLT-type scenarios ${ }^{3}$ [7], then it does not occur and the flux singularity remains unresolved.

\section{Spherical 5-branes}

Whenever one considers $p$-branes in background with $F_{6-p}$-flux there is the possibility that the $p$-branes polarise into a spherical $(p+2)$-brane carrying $(p+2)$-brane dipole charge and the original $p$-brane monopole charges induced by gauge fluxes on the worldvolume [31]. This possibility is enhanced by the flux singularity discussed in the previous section. In the case at hand one expects two polarisation channels: a spherical D5 wrapping a contractible $S^{2}$ inside the $M_{3}$ that is threaded by $H$ and a spherical NS5 wrapping a contractible $S^{2}$ inside the $S^{3}$ filled with $F_{3}$ (denoted $S_{F}^{3}$ ). If polarisation occurs, then the flux singularity is regularized in one of two ways: either the spherical branes sit at a finite distance from the original D-brane charge and the flux clumping parameter $\lambda$ takes a finite value throughout the solution, this is similar to what happened in [23], or the anti-D3 branes polarise to an NS5 brane wrapping an $S^{2}$ inside the $S^{3}$ with a runaway potential towards the other pole of the $S^{3}$. This latter possibility results in annihilation of flux against the anti-D3 brane charge as we will explain in more detail below.

In this paper we follow a procedure for computing the polarisation potentials that has become standard, but is not without danger of not being fully waterproof. The procedure aims to take into account the backreaction of the branes in computing the polarisation potential because some polarisation phenomena are invisible in the probe limit. Since the polarisation potential necessarily relies on probe brane actions the actual computation is in between probe and full backreaction. The idea is the following: if one wants to know whether $N$ anti-D3 branes polarise into D5 branes, one can work in the limit where $N-p$ antibranes backreact and $p$ are treated as probe. For large $N$ and with $p \ll N$ this should be a valid assumption. Hence one can use the probe action for $p$ D3 branes and study their polarisation process. A caveat could be that the probe calculation differs if instead of backreacting $N-p$ antibranes one uses the backreaction of spherical D5 branes carrying $N-p$ anti-D3 charges. If for instance the latter computation reveals a meta-stable position for polarisation of the $p$ probes one might take it as a sign that the full stack of D3 branes

\footnotetext{
${ }^{3}$ In KKLT type scenarios the CC can be decoupled from the KK scale due to orientifolds and quantum corrections.
} 
polarises into a meta-stable state. To our knowledge it has so far been consistent to work in the situation in which one regards the backreaction of the $N-p$ D3 branes. A rationale for this could be that if the $p$ D3 probes do not polarise into a meta-stable position when the antibranes backreact, then neither will they if one considers the backreaction of spherical D5 probes. The reason is that one could think of the polarisation to proceed stepwise. We nonetheless emphasise that this should be better understood.

Finally we mention that for a compact solution, $M_{3}$ takes the form of a three-sphere which we denote by $S_{H}^{3}$. S-duality interchanges the roles of D5 and NS5 as well as the fluxes $F_{3}$ and $H$. So performing an S-duality effectively smears the D3 branes over $S_{H}^{3}$ and localises them inside $S_{F}^{3}$. We therefore expect the fully localised solution (that is localised on $\left.S_{H}^{3} \times S_{F}^{3}\right)$ to polarise into a web of $(p, q)$ 5-branes as in [27]. In what follows we discuss both channels.

\subsection{D5 polarisation}

There are generically two ways of showing that a $\mathrm{D} p$-brane polarizes to a $\mathrm{D}(p+2)$-brane; either by considering the non-abelian $\mathrm{D} p$-brane action as in [31], or by considering the probe action of a $\mathrm{D}(p+2)$-brane in the $\mathrm{D} p$-brane background [23]. In this paper we take the latter approach. The required terms of the D5-brane action in Einstein frame are

$$
S_{\mathrm{D} 5}=\mu_{5} \int\left\{-\mathrm{e}^{-\phi} \sqrt{-\operatorname{det}\left(\mathrm{e}^{\phi / 2} G-\mathcal{F}\right)}-C_{6}+\mathcal{F} \wedge C_{4}\right\},
$$

where $\mathcal{F}=B-F$. We will take $F=n \pi \mathrm{vol}_{2}$ and expand the D5-brane action for large $n$ for which polarisation is preferred and then look at the behaviour close to $\rho=0$. Instead of working through the computation here, we state the result and in section 4.3 we present a more general computation for $D p$-branes polarising into $D(p+2)$-branes, which includes this case by putting $p=3$. For $\mathrm{AdS}_{4}$ external space we find

$$
V \propto\left(4 \Lambda+\frac{1}{2} k_{0}^{2}\right) \bar{\rho}^{2}-2 k_{0} \bar{\rho}^{3}+3 \bar{\rho}^{4},
$$

where $\bar{\rho}$ is a dimensionless distance from the D3 branes and is defined in section 4.3. The cosmological constant $\Lambda$ is normalized to -3 for AdS external space and vanishes of course if the external space is flat. The numerical value $k_{0}$ is directly related to $\Lambda$ but with a proportionality factor that depends on the details of the solution. We can however estimate the value of $k_{0}$ by smearing for which we find $k_{0}=\sqrt{6}$ (cf. section 4.3) and the potential takes the form in figure 1.

What we find is that the D5 polarisation occurs for the AdS solutions, but that it cannot happen for the non-compact Minkowski solutions. This is in perfect agreement with what has been found for the D6 solutions of [1,9] as shown in $[2,24]$ (see also [1, 11]).

The polarisation into (meta-)stable spherical D5 branes smoothens out the singular pile-up of the three-form fluxes. In other words, $\lambda$ remains finite throughout the solution. The only singularities are the expected ones in the metric and form fields that comes from the localised charge and tension of (spherical) branes. This smoothening can be deduced quite easily by repeating the computation of the size of $\lambda$ at the boundary of the spherical 


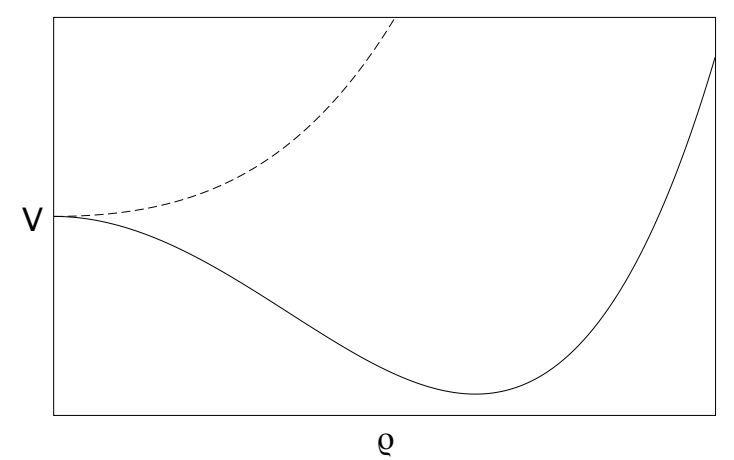

Figure 1. The potential $V$ (3.2) for probe D5 branes in the background of D3 branes. The dashed line shows the polarisation potential when the external space is flat while the solid line displays the potential for AdS space, for which the probes are tachyonic near $\bar{\rho}=0$. A stable state exists at a finite distance away from the tip.

D5 brane. One simply needs to use the near-brane expansion of a 5-brane that is smeared over 3-directions. This computation is completely analogues to what has been done in [24] (equations (2.16) and (2.17)).

\subsection{NS5 polarisation and flux decay}

Our $\mathrm{AdS}_{4}$ solution is constructed by smearing the D3 branes over $S_{F}^{3}$ which makes it unclear how brane polarisation can proceed inside $S_{F}^{3}$. For the polarisation into D5 branes to occur it was necessary to have a solution localised in $M_{3}$ because the localised solution backreacts in such a way that the profiles of the background supergravity fields induce a minimum in the D5 polarisation potential at a finite size for the spherical 5-brane. But the "dual" channel does not need localised branes, one just has to keep in mind that the brane polarisation computation is a probe computation and probes are localised. It turns out that the probe computation parallels those done in $[6,13,19])$.

Whereas the physics of the D5 polarisation channel is to resolve the flux clumping, the physics of the NS5 channel is brane-flux decay [6]. If the D3 branes polarise into NS5 branes that either tunnel or move perturbatively to the North Pole of the $S_{F}^{3}$ one can verify that its monopole charge has shifted to $M-p$ instead of $-p$ D3 charges. The interpretation of this is that $M$ units of 3-form flux materialised into $M$ physical D3 branes that consequently annihilate with the $p$ anti-D3 branes to leave $M-p$ D3 branes in the process.

The NS5 potential is calculated by considering the worldvolume action of an NS5 brane (treated as a probe) in the background of D3 branes and fluxes. The main difference to the computation done in [6] has to do with the Wess-Zumino (WZ) action for the NS5 brane. The complete NS5 brane action for $C_{0}=0$ takes the form ${ }^{4}$

$$
-\mu_{\mathrm{NS} 5} \int \mathrm{e}^{-2 \phi} \sqrt{-\operatorname{det}\left(\mathrm{e}^{\phi / 2} G-\mathrm{e}^{\phi} \mathcal{F}\right)}+\mu_{\mathrm{NS} 5} \int\left(B_{6}+\mathcal{F} \wedge C_{4}\right)
$$

\footnotetext{
${ }^{4}$ The sign if the WZ action is fixed by demanding that a spherical NS5 brane induces a D3 brance charge, i.e. that the sign of the $\int F \wedge C_{4}$ matches the sign of the WZ action for the D3 branes above.
} 
The world volume field strength $F$ enters through the combination $\mathcal{F}=F-C_{2}$. To evaluate the WZ action we need to find $B_{6}$. We have parametrized $H$ as $-\lambda \mathrm{e}^{\phi} \star_{6} F_{3}$ and the dual flux is defined as $H_{7}=\mathrm{e}^{-\phi} \star H$. The gauge potential for $H_{7}$ can be defined through the Bianchi identity $\mathrm{d} H_{7}=-F_{5} \wedge F_{3}$. Evaluating the right hand side for our Ansatz we determine

$$
H_{7}=\mathrm{d} B_{6}-C_{4} \wedge F_{3} .
$$

From this we find $B_{6}$ by writing

$$
\mathrm{d} B_{6}=\mathrm{e}^{-\phi} \star_{10} H+C_{4} \wedge F_{3}=\left(\lambda \mathrm{e}^{4 A}-\alpha+\alpha_{0}\right) \operatorname{vol}_{4} \wedge F_{3}=\alpha_{0} \operatorname{vol}_{4} \wedge F_{3},
$$

such that

$$
B_{6}=\alpha_{0} \operatorname{vol}_{4} \wedge C_{2},
$$

which implies that the WZ Lagrangian takes the form

$$
B_{6}+\mathcal{F} \wedge C_{4}=\left(\alpha C_{2}-\left(\alpha-\alpha_{0}\right) F\right) \wedge \operatorname{vol}_{4} .
$$

We also need an expression for $C_{2}$ that correctly reproduces the constant $F_{3}$-flux via $F_{3}=\mathrm{d} C_{2}$

$$
C_{2}=\frac{M}{2}\left(\psi-\frac{1}{2} \sin (2 \psi)\right) \operatorname{vol}_{2}
$$

where $\psi$ is used as the third Euler angle on the smeared three-sphere. Remember that the D3 branes are localised on $M_{3}$ at $\rho=0$ (cf. equation (2.1)). The polarisation potential depends on $\psi$ but takes a different form depending on the position on $M_{3}$. We will denote the potential by $V_{\rho}(\psi)$. Finally we let $F_{2}=\pi p$ vol $_{2}$ where $p$ sets the D3 brane charge of the probe. The full polarisation potential is obtained by dividing the NS5 action by $\left(-\mu_{\mathrm{NS} 5} M\right)$ and relevant volume factors, and the result is

$$
\begin{aligned}
V_{\rho}(\psi)= & \mathrm{e}^{4 A} \sqrt{\frac{1}{M^{2}} \mathrm{e}^{4 B-\phi} \sin ^{4} \psi+\frac{1}{4}\left(2 \pi \frac{p}{M}-\psi+\frac{1}{2} \sin (2 \psi)\right)^{2}} \\
& -\frac{\alpha}{2}\left(\psi-\frac{1}{2} \sin (2 \psi)\right)-\pi\left(\alpha-\alpha_{0}\right) \frac{p}{M}
\end{aligned}
$$

This potential is valid for either the AdS or flat D3 brane solutions discussed above. The main difference when analysing the potential lies in the fact that the D3 branes in AdS polarize immediately to D5 branes.

Let us discuss the potential in the flat case for which we have shown that the D3 branes do not polarise into D5 branes. Even though $\mathrm{e}^{4 A}$ vanishes as $\rho \rightarrow 0, \alpha$ stays constant. This means that the first term in (3.9) vanishes as $\rho \rightarrow 0$ but the second term does not. The potential therefore reduces to

$$
V_{\rho=0}(\psi)=-\frac{\alpha_{0}}{2}\left(\psi-\frac{1}{2} \sin (2 \psi)\right) .
$$

The number $\alpha_{0}$ is finite and positive, just as for the compact AdS solution. This can be understood from studying the $F_{5}$ Bianchi identity which leads to a strong constraint 


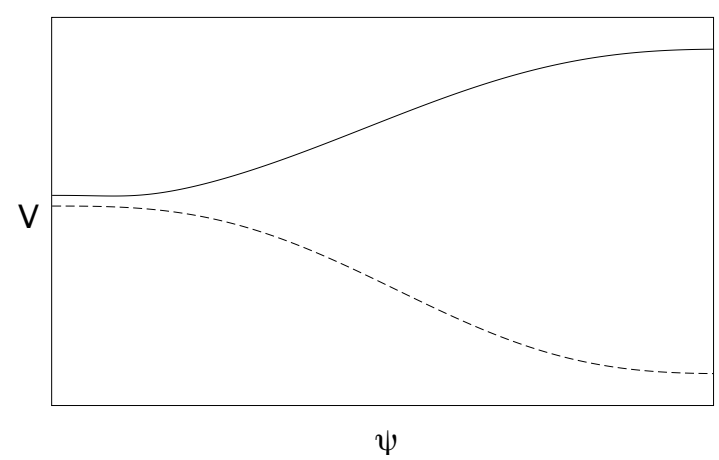

Figure 2. The polarisation potential for spherical NS5 branes. The dashed line is a plot of the potential (3.9) for flat external space and at $\rho=0$. The solid line is evaluated for AdS external space with $\lambda_{\max }=1 / 2$.

on the form of $\alpha$ when combined with D3 brane boundary conditions and the asymptotic behaviour of the fields far away from the D3s. The analysis is completely analogous to the one done in [10] in the case of D6 branes. Hence the NS5 potential for flat branes indicate a perturbative brane-flux decay as shown in figure 2. Note that the potential (3.10) is completely independent of the ratio $p / M$, this shows that no matter how small the $\overline{\mathrm{D} 3}$ charge is carried by the probe, the NS5-brane will always be pushed away by the divergent $H$-flux at the tip $\rho \rightarrow 0$.

For an $\mathrm{AdS}_{4}$ external space the situation is different, the polarisation of the D3 branes to D5's regularizes the singularity in $\lambda$ such that the both terms in equation (3.9) play a role. Brane-flux decay depends on the relative size of the two terms in the potential. The first term effectively pulls the NS5 probe towards the D5 branes while the second term pushes the probe away. We have seen that for the fully smeared solution brane-flux decay cannot occur for a very simple reason; the energy of the vacuum would increase. We expect the same result to hold in the partially localised case (and in the fully localised case). In order to confirm this we would have to scan the potential $V_{\rho}$ for all values of $\rho$ and show that the gravitational pull of the first term outweighs the electromagnetic push of the second one. It would then be enough to find an upper bound on the function $\lambda$ and show that it is less than the smeared value $\lambda=1$. For the AdS vacua, we have plotted the expected qualitative behavior for the NS5 potential in figure 2 .

\section{Anti-D $p$ solutions}

The D3 brane solutions discussed so far belongs to a class of $\mathrm{D} p$-brane solutions studied first in [17], where the smeared limit and flat limit were explored. Here we study the setup in more generality.

The metric takes the form

$$
\mathrm{d} s^{2}=\mathrm{e}^{2 A} \mathrm{~d} s_{\mathrm{AdS}_{p+1}}^{2}+\mathrm{e}^{2 B} \mathrm{~d} s_{S^{6-p}}^{2}+\mathrm{e}^{2 C} \mathrm{~d} s_{M_{3}}^{2}, \quad \text { and } \quad \mathrm{d} s_{M_{3}}^{2}=\mathrm{d} \rho^{2}+\mathrm{e}^{2 D} \mathrm{~d} \Omega^{2}
$$


where $\mathrm{d} s_{\mathrm{AdS}_{p+1}}^{2}, \mathrm{~d} s_{S^{6-p}}^{2}$ and $\mathrm{d} \Omega^{2}$ are the metrics for $\mathrm{AdS}_{p+1}$, the $(6-p)$-sphere $S^{6-p}$ and the two-sphere $S^{2}$ respectively. Once again we choose the metrics such that the corresponding unwarped Ricci tensors take the canonical form

$$
\hat{R}_{\mu \nu}=\Lambda \hat{g}_{\mu \nu}, \quad \hat{R}_{m n}=(5-p) \hat{g}_{m n}, \quad \hat{R}_{i j}=\hat{g}_{i j} .
$$

The hatted variables denote the unwarped quantities, and

$$
\mathrm{d} s_{\mathrm{AdS}_{p+1}}^{2}=\hat{g}_{\mu \nu} \mathrm{d} x^{\mu} \mathrm{d} x^{\nu}, \quad \mathrm{d} s_{S^{6-p}}^{2}=\hat{g}_{m n} \mathrm{~d} x^{m} \mathrm{~d} x^{n}, \quad \mathrm{~d} \Omega^{2}=\hat{g}_{i j} \mathrm{~d} x^{i} \mathrm{~d} x^{j} .
$$

The warp factors $A, B, C$ and $D$ are only functions of the coordinate $\rho$ and so the Einstein equations reduce to ordinary differential equations for the warp factors. We parametrize our fluxes as follows:

$$
\begin{aligned}
H & =-\lambda \mathrm{e}^{\frac{p+1}{4} \phi} \star_{9-p} F_{6-p}, \\
F_{6-p} & =M \operatorname{vol}_{S^{6-p}}, \\
F_{8-p}^{\perp} & =\mathrm{e}^{-(p+1) A-\frac{p-3}{2} \phi} \star_{9-p} \mathrm{~d} \alpha .
\end{aligned}
$$

The functions $\lambda$ and $\alpha$ as well as the dilaton are assumed, like the warp factors, to depend only on the $\rho$ coordinate. The volume form $\operatorname{vol}_{6-p}$ is the unwarped volume form on the $(6-p)$-sphere. The superscript $\perp$ on $F_{8-p}$ indicates the fact that we are specifying the components transverse to the brane worldvolume. For $p=3$ the components of $F_{5}$ along the worldvolume are also non-zero by the selfduality condition. From the equation of motion for $H$ we determine

$$
\alpha=-(-1)^{p} \lambda \mathrm{e}^{(p+1) A+\frac{p-3}{4} \phi}=-(-1)^{p} \lambda \beta,
$$

where the function $\beta$ is defined by the second equality. The only unsatisfied form field equation is the $F_{8-p}$ Bianchi identity, which takes the form

$$
\mathrm{d} F_{8-p}-H \wedge F_{6-p}=N_{\mathrm{D} p} \mu_{p} \delta_{9-p}
$$

\subsection{The AdS curvature}

Once again we can relate the zero point value of the function $\alpha$ to the AdS curvature via the relation given in [15]. For the Ansatz specified above we find

$$
\frac{p-1}{2} \Lambda=\frac{1}{4 V_{p+1}}\left[N_{\mathrm{D} p} S_{\mathrm{loc}}+\frac{1}{V_{9-p}} \int H \wedge\left(\mathrm{e}^{-\phi} \star_{10} H-\sigma\left(F_{6-p}\right) \wedge C_{p+1}\right)\right],
$$

from which we can get rid of the first term by a gauge choice for $C_{p+1}$ along the worldvolume:

$$
C_{p+1}^{\|}=-\left(\alpha-\alpha_{0}\right) \sigma\left(\operatorname{vol}_{p+1}\right)
$$

where $\alpha_{0}$ is the value of $\alpha$ at $\rho=0$. Putting this together we get

$$
\frac{p-1}{2} \Lambda=\frac{\alpha_{0}}{4 V_{9-p}} \int H \wedge F_{6-p}=-\frac{\alpha_{0} Q}{4 V_{9-p}},
$$


where we used equation (4.6) to evaluate the integral and

$$
V_{9-p}=\int \star_{9-p} \mathrm{e}^{(p-1) A} .
$$

Since the warped volume $V_{9-p}$ is not known a priori, the equation (4.9) does not fix the value of $\alpha_{0}$. However, we now know that for non-vanishing cosmological constant, the value of $\alpha_{0}$ must be a strictly positive number. This fact enables us to conclude that a singularity is developed in the energy density of $H$ just as for the special case $p=3$ discussed above. Since the argument is completely analogous we do not repeat it here.

Concerning supersymmetry we explain in appendix $\mathrm{C}$ that compact $\mathrm{AdS}_{p}$ solutions cannot be supersymmetric for $p=3,4,5$, when an anti- $\mathrm{D} p$ singularity is assumed at $\rho=0$.

\subsection{Flat solutions}

As with the anti-D3 branes, we can also investigate flat solutions, or solutions with a nonzero CC, but whose value is not fixed by compactness and hence decoupled from the scale set by the brane charges and the fluxes. The metric Ansatz is simply the generalisation of the Ansatz used for anti-D3 branes:

$$
\mathrm{d} s^{2}=\mathrm{e}^{2 A} \mathrm{~d} s_{\text {Mink }_{p+1}}^{2}+\mathrm{e}^{2 B} \mathrm{~d} s_{\mathbb{T}^{6-p} / S^{6-p}}^{2}+\mathrm{e}^{2 C} \mathrm{~d} s_{M_{3}}^{2}, .
$$

These solutions describe anti-Dp branes smeared over the $\mathbb{T}^{6-p} / S^{6-p}$. The solutions with the torus factor can be obtained from T-duality of the anti-D6 solution [10].

\subsection{Brane polarisation}

We now compute the potential for a probe $\mathrm{D}(p+2)$-brane in the background of $N_{\mathrm{D} p}$ $\mathrm{D} p$-branes. This is a standard computation that we repeat for completeness and we find agreement with the results of $[2,24]$ for $p=6$ and [25] for $p=3$.

The probe action in this case is

$$
S_{\mathrm{D}(p+2)}=-\mu_{p+2} \int\left\{\mathrm{e}^{-\phi} \sqrt{-\operatorname{det}\left(\mathrm{e}^{\phi / 2} G-\mathcal{F}\right)}-(-1)^{p} \sigma\left(C_{p+3}-\mathcal{F} \wedge C_{p+1}\right)\right\},
$$

where as before $\mathcal{F}=B-F$ and $F$ is the world volume field strength. We take $F=\pi n \mathrm{vol}_{2}$ and expand the action for large $n$ to obtain

$$
V \propto(\pi n-b) L_{\mathrm{D} p}+(-1)^{p} \gamma+\frac{\beta \mathrm{e}^{\phi+4 C+4 D}}{2(\pi n-b)},
$$

where

$$
B=b(\rho) \operatorname{vol}_{2}, \quad C_{p+3}=\gamma(\rho) \sigma\left(\operatorname{vol}_{p+1}\right) \wedge \operatorname{vol}_{2},
$$

and

$$
L_{\mathrm{D} p}(\rho)=\beta-(-1)^{p}\left(\alpha-\alpha_{0}\right)=\beta(1+\lambda)+(-1)^{p} \alpha_{0} .
$$

The functions $b(\rho)$ and $\gamma(\rho)$ are determined by employing the definition of $H$ and $F_{p+4}$ in terms of their potentials and comparing to the anzats. The result is

$$
\begin{aligned}
b^{\prime}(\rho) & =M \alpha \mathrm{e}^{\phi-(p+1) A-(6-p) B+3 C+2 D}, \\
\gamma^{\prime}(\rho) & =\left(\beta^{2}-\alpha\left(\alpha-\alpha_{0}\right)\right) M \mathrm{e}^{\phi-(p+1) A-(6-p) B+3 C+2 D} .
\end{aligned}
$$


We will use these equations to determine the behaviour of $b$ and $\gamma$ close to the $\mathrm{D} p$-branes. Before we expand the fields we present a differential equation for $L_{\mathrm{D} p}$ which is obtained by combining the external Einstein equation with the dilaton equation and the Bianchi identity (4.6). The equation is remarkably simple, and in particular no source terms appear:

$$
\nabla^{2} L_{\mathrm{D} p}-\beta^{-1}\left(\nabla L_{\mathrm{D} p}\right)^{2}=\beta\left[(p+1) \Lambda \mathrm{e}^{-2 A}+\frac{(1+\lambda)^{2}}{2} \mathrm{e}^{\frac{p-1}{2} \phi}\left|F_{6-p}\right|^{2}\right] .
$$

We are now in position to expand the fields close to the $\mathrm{D} p$-branes so as to obtain an expression for the potential $V$ close to the branes. We use the standard boundary conditions of the fields close to a $\mathrm{D} p$ brane. This is the expected behaviour based for example on the analysis in [10]

$$
\begin{aligned}
\mathrm{e}^{2 A} & \approx \rho^{\frac{7-p}{8}}\left(a_{0}+a_{1} \rho\right), \\
\mathrm{e}^{2 B} & \approx \rho^{\frac{-1-p}{8}}\left(b_{0}+b_{1} \rho\right), \\
\mathrm{e}^{2 C} & \approx \rho^{\frac{-1-p}{8}}\left(c_{0}+c_{1} \rho\right), \\
\mathrm{e}^{2 D} & \approx \rho^{2}\left(1+d_{1} \rho\right), \\
\mathrm{e}^{2 \phi} & \approx \rho^{\frac{p-3}{2}}\left(f_{0}+f_{1} \rho\right), \\
L_{\mathrm{D} p} & \approx \rho\left(l_{0}+\rho l_{1}\right),
\end{aligned}
$$

the last expansion in this list is determined by noting that we chose the gauge for $C_{p+1}$ such that the constant part of $L_{\mathrm{D} p}$ vanishes. The constants $a_{0}, b_{0}, c_{0}$ and $f_{0}$ can be rewritten in terms of the number of Dp branes $N_{\mathrm{D} p}$ and string coupling $g_{s}$ by studying the flat $p$-brane solutions. The near brane behaviour of $\alpha$ is given by

$$
\alpha \approx \alpha_{0}+\rho \alpha_{1}
$$

Below we need the first term in the expansion of $\beta$

$$
\beta \approx \rho \beta_{0}=\rho f_{0}^{\frac{p-3}{8}} a_{0}^{\frac{p+1}{2}} .
$$

Expanding the equation (4.17) to leading order we immediately find $l_{0}=0$ which implies

$$
\beta_{0}=(-1)^{p} \alpha_{1}
$$

The next order coefficient is

$$
6 l_{1}=\frac{c_{0} \beta_{0}}{a_{0}}\left((p+1) \Lambda+\frac{1}{2}\left(\lambda_{0} \beta_{0} M\right)^{2} f_{0}^{\frac{1}{2}} a_{0}^{-p} b_{0}^{p-6}\right) .
$$

Expanding the gauge potentials gives

$$
\begin{aligned}
& b(\rho) \approx \frac{1}{2} \rho^{2} \alpha_{0} M f_{0}^{\frac{1}{2}} a_{0}^{-\frac{p+1}{2}} b_{0}^{-\frac{6-p}{2}} c_{0}^{\frac{3}{2}}, \\
& \gamma(\rho) \approx-\frac{1}{3} \rho^{3} \alpha_{0} \alpha_{1} M f_{0}^{\frac{1}{2}} a_{0}^{-\frac{p+1}{2}} b_{0}^{-\frac{6-p}{2}} c_{0}^{\frac{3}{2}} .
\end{aligned}
$$


Using these results it is straightforward to write down the first few terms of the expansion of the brane potential,

$$
V \approx \frac{\beta_{0}(\pi n)^{3}}{6 a_{0}^{2} f_{0}^{\frac{1}{2}}}\left\{\left((p+1) \Lambda+\frac{1}{2} k_{0}^{2}\right) \bar{\rho}^{2}-2 k_{0} \bar{\rho}^{3}+3 \bar{\rho}^{4}\right\},
$$

where

$$
\bar{\rho}=\frac{\sqrt{c_{0} a_{0}} f_{0}^{\frac{1}{4}}}{\pi n} \rho \quad \text { and } \quad k_{0}=\left(\alpha_{0} M\right) a_{0}^{-\frac{p}{2}} b_{0}^{-\frac{6-p}{2}} f_{0}^{\frac{1}{4}} .
$$

We now see that the polarisation potential (4.24) is fully determined as soon as the constant $k_{0}$ is given.

Besides the extremum at the origin, this potential has at most two more extrema at

$$
\bar{\rho}=\frac{k_{0}}{2} \pm \sqrt{-\frac{k_{0}^{2}}{12}-\frac{(p+1) \Lambda}{3}},
$$

which shows that polarisation occurs in AdS space when

$$
-\Lambda \geq \frac{k_{0}^{2}}{4(p+1)} .
$$

One of our key results is that polarisation for solutions where the CC is parametrically small is impossible. This in particular includes the flat solutions which obviously do not polarise by equation (4.26).

Let us now estimate $k_{0}$ for the AdS solutions. The second-order equations of motion can unfortunately not help without solving the system completely. We can however estimate $k_{0}$ by solving the equation (4.17) in the fully smeared limit. This amounts to putting the derivatives to zero and replacing $\lambda$ with its smeared value [17]

$$
\lambda \rightarrow \frac{p-1}{2}
$$

We then find

$$
\frac{1}{2} k_{0}^{2} \rightarrow \frac{p(p-1)^{2}}{p+1}
$$

which can be inserted into the polarisation potential (4.24). The potential is easily verified from equation (4.27) to allow for a polarisation at a finite value of $\bar{\rho}$.

In this section we used two expansions, one for large $n$ and another for small radius $\rho$. Clearly we can choose $\rho$ small to justify the near brane expansion and $N_{\mathrm{D} p}$ large to stay within the probe approximation, $n \ll N_{\mathrm{D} p}$. Within this regime a minimum of the $\mathrm{D}(p+2)$ potential can be deduced in the following way. Since the WZ term becomes less important when we move away from the anti-D $p$, the DBI term dominates forcing the potential upward. By checking whether the $\mathrm{D}(p+2)$ potential decreases away from the point $\rho=0$ we deduce the existence of a minimum. Obviously, a more careful analysis is needed to quantitatively trust the calculation from the previous section up to the minimum of the potential but this is unnecessary to verify brane polarisation. 


\section{Conclusion}

We have shown that there is a consistent picture for the flux singularities associated with anti- $\mathrm{D} p$ solutions that are smeared over $6-p$ compact directions. These solutions come in two types: compact $\mathrm{AdS}_{p+1}$ solutions and non-compact flat solutions and both feature singular fluxes that partially screen the antibrane charges. We have found a story similar to what happened for localised anti-D6 branes $[1,2,24]$ : the flat solutions do not polarise into spherical branes whereas the AdS solutions do. As a consequence the AdS solutions have regular flux clouds in the supergravity limit. Compact AdS vacua have a cosmological constant related to the energy in such a way that brane-flux decay would increase the energy. This is opposite to the flat solution, where brane-flux decay lowers the energy. So either flux clouds that are singular at the SUGRA level initiate perturbative brane-flux decay $[13,19]$ or brane polarisation has to occur in order to resolve the flux singularity. We have found exactly that. Concerning the flat solutions we also find consistency with $[13,19]$ : the flux clumping is too large and causes perturbative brane-flux decay such that the smooth solution is expected to be time dependent. This is in agreement with the absence of regular finite temperature solutions [16, 20-22].

An important restriction of our work is the smearing of the antibranes over the internal $S^{6-p}$ or $\mathbb{T}^{6-p}$. It is important to investigate brane polarisation and brane-flux decay for fully localised branes. This becomes especially important in case one looks at backgrounds with very few, or even a single, SUSY-breaking antibrane. For a single antibrane it is clearly not physical to smear the charges over a compact submanifold and it is exactly in the regime of a single brane that it has been argued that the meta-stable states are most likely to exist $[6,14]$. An important argument here in favor of meta-stability is that the flux clumping effects of $[13,19]$ are only relevant at the South Pole of the $S^{3}$ and once the NS5 brane moves away from the South Pole the forces that push it over the equator are diluted $^{5}$ as depicted in figure 3 .

At first sight this allows a classical barrier against brane-flux decay and would seem consistent with a recent claim that regular finite temperature solutions can exist if the antibranes are fully localised [22]. If this is the case, it is a good indication that there is no tachyonic mode that describes the onset of brane-flux decay and it would therefore be most important to find a full proof of the claims in [22].

The sensitivity of antibrane uplifting to instabilities arises through the use of a warped throat that redshifts string-scale energies down to much smaller energy scales. This locally creates a lack of scale-separation and various modes can mix with Kaluza-Klein (KK) modes that become light [32]. The flux-clumping instabilities, if present, are an example of this effect since flux gradients correspond to KK modes and mix with the modes that correspond to the NS5 position. Checking full stability remains therefore a subtle issue for scenarios that are based on antibrane uplifting. One could for instance worry about the tachyonic modes found in [33] or even Gregory-Laflamme-like instabilities in the screened anti-D3 brane [34].

\footnotetext{
${ }^{5}$ We like to thank U. Danielsson, D. Junghans and the authors of [14] for discussions on that matter.
} 

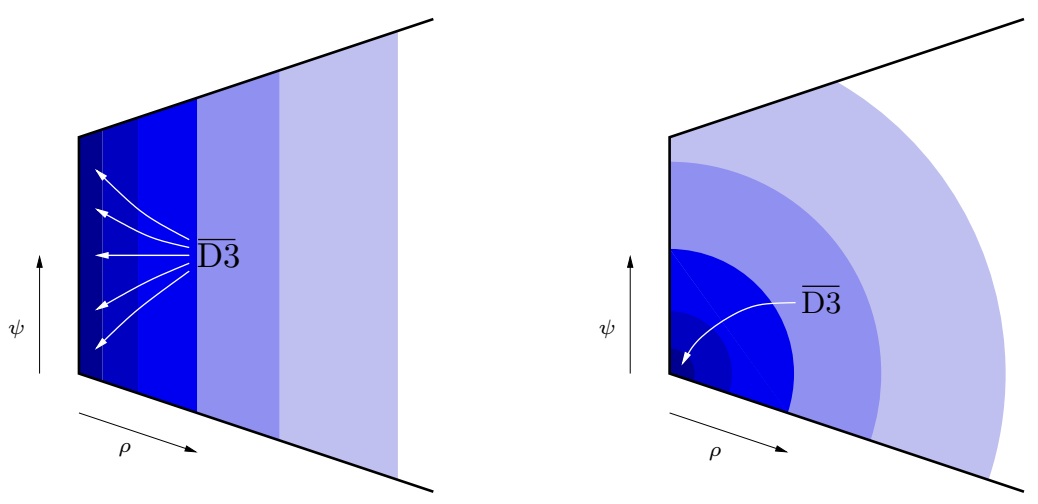

Figure 3. The difference between the flux clumping between antibranes smeared over the tip of the KS throat (left) and antibranes localised at the South Pole of the tip (right). At every point in the $\psi$-direction we suppressed the two-sphere (with zero size at South and North Pole, $\psi=0, \pi$, and maximum size in between). The flux clumping on the right is less severe in the middle of the $\psi$ direction, such that the force that pushes the NS5 towards the North Pole is less and could be small enough to create a classical barrier.

Finally, there is a black hole analogue to antibrane SUSY breaking in flux backgrounds, which are near-extremal micro-state geometries build from meta-stable supertubes [35, 36]. One could worry whether these constructions also feature the problem of enhanced decay due to flux-clumping like effects. We have good reasons to believe this is not the case and hope to report on this in the near future.

\section{Acknowledgments}

We would like to thank Iosif Bena, Ulf Danielsson, Alessandro Tomasiello, Daniel Thompson, Joseph Polchinski, Andrea Puhm and especially Nikolay Bobev for useful discussions. TVR is supported by a Odysseus grant nr. G.0.E52.14N and a Pegasus fellowship of the FWO. BT is aspirant FWO. We also acknowledge support from the European Science Foundation Holograv Network.

\section{A Notation and conventions}

The bulk type II action takes the form

$$
S=\int \star_{10}\left\{R-\frac{1}{2}|\mathrm{~d} \phi|^{2}-\frac{1}{2} \mathrm{e}^{-\phi}|H|^{2}-\frac{1}{4} \sum_{n} \mathrm{e}^{\frac{5-n}{2} \phi}\left|F_{n}\right|^{2}\right\},
$$

where $R$ is the curvature scalar of the Einstein frame metric $G$ with mostly plus signature. The kinetic terms for the dilaton $\phi$, the NSNS 3-form $H$ and the RR forms $F_{n}$ are written using the short hand notation

$$
\left|\omega_{p}\right|^{2} \star_{10} 1=\star_{10} \omega_{p} \wedge \omega_{p}=\frac{1}{p !} G^{M_{1} N_{1}} \cdots G^{M_{p} N_{p}} \omega_{M_{1} \cdots M_{p}} \omega_{N_{1} \cdots N_{p}} \star_{10} 1
$$


where

$$
\omega_{p}=\frac{1}{p !} \omega_{M_{1} \cdots M_{p}} \mathrm{~d} X^{M_{1}} \wedge \cdots \wedge \mathrm{d} X^{M_{p}}
$$

is any $p$-form. Notice that we are using the democratic formulation of [37] which means that all RR field strengths $F_{n}$ with $n=1,3,5,7$ and 9 in type IIB and $n=0,2,4,6,8$ and 10 in type IIA appear in the action. In this way the bulk action does not contain any Chern-Simons terms but a duality relation between the RR fields,

$$
\mathrm{e}^{\frac{5-n}{2} \phi} F_{n}=\star_{10} \sigma\left(F_{10-n}\right),
$$

must be imposed on-shell by hand. The reversal operator $\sigma$ has been introduced to simplify many equations in the paper, it does only introduce a sign depending on the degree of the form it acts on, i.e.

$$
\sigma\left(\omega_{p}\right)=(-1)^{\frac{p(p-1)}{2}} \omega_{p}
$$

Including the localized action for an anti-D $p$ brane

$$
S_{\mathrm{loc}}=-\mu_{p} \int_{N_{p+1}}\left\{\mathrm{e}^{\frac{p-3}{4} \phi} \star_{p+1} 1+(-1)^{p} \sigma\left(C_{p+1}\right)\right\}
$$

we see that the Bianchi identity for $F_{8-p}$, the Einstein equation and the dilaton equation acquire correction due to the presence of the branes. In the localized action we have already made use of the fact that the worldvolume field strength $\mathcal{F}$ vanishes in the setup we consider. The fields appearing in the D-brane action are understood as the pull-backs of their bulk counterparts. For reference we present the modified Bianchi identity for $F_{8-p}$

$$
\mathrm{d} F_{8-p}-H \wedge F_{6-p}=\mu_{p} \delta_{9-p}\left(N_{p+1}\right),
$$

where $\delta_{9-p}\left(N_{p+1}\right)$ denotes the $(p+1)$-form with delta distribution support on the brane worldvolume, i.e.

$$
\delta_{9-p}\left(N_{p+1}\right)=\star_{9-p} 1 \delta\left(N_{p+1}\right) .
$$

\section{B Second-order equations}

In this appendix we present the second-order differential equations for the Ansatz in section 2. The $F_{5}$ Bianchi identity implies

$$
\left(\alpha^{\prime} e^{3 B+C+2 D-4 A}\right)^{\prime}=\lambda M^{2} e^{\phi-3 B+3 C+2 D}-N_{\mathrm{D} 3} \mu_{3} \delta_{6} .
$$

We use a prime to denote a derivative with respect to $\rho$. The dilaton equation gives

$$
\phi^{\prime \prime}+(4 A+3 B+C+2 D)^{\prime} \phi^{\prime}=\frac{1}{2} M^{2} e^{\phi+2 C-6 B}\left(1-\lambda^{2}\right) .
$$

We present the Einstein equation in the trace reversed form

$$
R_{M N}=\hat{T}_{M N}
$$


The Ricci tensor is

$$
\begin{aligned}
R_{\mu \nu}= & -e^{-2 C}\left(4 e^{2 A} A^{2}+e^{2 A}\left(3 B^{\prime}+C^{\prime}+2 D^{\prime}\right) A^{\prime}+3 e^{2 C}+e^{2 A} A^{\prime \prime}\right) e^{-2 A} g_{\mu \nu}, \\
R_{i j}= & e^{-2 C}\left(-3 e^{2 B} B^{\prime 2}-4 e^{2 B} A^{\prime} B^{\prime}-e^{2 B}\left(C^{\prime}+2 D^{\prime}\right) B^{\prime}+2 e^{2 C}-e^{2 B} B^{\prime \prime}\right) e^{-2 B} g_{i j}, \\
R_{\rho \rho}= & -4 A^{\prime 2}+4 C^{\prime} A^{\prime}-3 B^{\prime 2}-2 D^{\prime 2}+3 B^{\prime} C^{\prime}-2 C^{\prime} D^{\prime} \\
& -4 A^{\prime \prime}-3 B^{\prime \prime}-2 C^{\prime \prime}-2 D^{\prime \prime} \\
R_{a b}= & \left(1-e^{2 D}\left(C^{\prime}+D^{\prime}\right)^{2}-4 e^{2 D} A^{\prime}\left(C^{\prime}+D^{\prime}\right)-3 e^{2 D} B^{\prime}\left(C^{\prime}+D^{\prime}\right)\right. \\
& \left.-e^{2 D}\left(D^{\prime 2}+C^{\prime} D^{\prime}+C^{\prime \prime}+D^{\prime \prime}\right)\right) e^{-2 C-2 D} g_{a b} .
\end{aligned}
$$

The components of the trace-reversed energy-momentum tensor are

$$
\begin{aligned}
& \hat{T}_{\mu \nu}=\frac{-1}{8} g_{\mu \nu}\left(\left(1+\lambda^{2}\right) M^{2} e^{\phi-6 B}+2 e^{-8 A-2 C}\left(\alpha^{\prime 2}\right)+2 \mu_{3} N \delta\right), \\
& \hat{T}_{i j}=\frac{1}{8} g_{i j}\left(M^{2}\left(3-\lambda^{2}\right) e^{\phi-6 B}+2\left(\alpha^{\prime}\right)^{2} e^{-8 A-2 C}\right) \\
& \hat{T}_{\rho \rho}=\frac{1}{2} \phi^{\prime 2}+\frac{1}{8} e^{2 C+\phi-6 B}\left(3 \lambda^{2}-1\right) M^{2}-\frac{1}{4}(\alpha)^{\prime 2} e^{-8 A} \\
& \hat{T}_{a b}=\frac{1}{8} g_{a b}\left(M^{2}\left(3 \lambda^{2}-1\right) e^{\phi-6 B}+2\left(\alpha^{\prime}\right)^{2} e^{-8 A-2 C}\right) .
\end{aligned}
$$

\section{Supersymmetric AdS}

The BPS-equations for our AdS solutions (4.1)-(4.4) are:

$$
\begin{aligned}
16(\nabla \phi)^{2}= & -\gamma^{-2} M^{2}\left(4 \beta^{-2} \alpha^{2}-(p-1)^{2}\right) \\
& +(p-3)^{2} \beta^{-2}(\nabla \alpha)^{2}, \\
(\nabla(4 A+\phi))^{2}= & -16 e^{-2 A}+\left[\gamma^{-2} M^{2}+\beta^{-2}(\nabla \alpha)^{2}\right] \\
\left(4 \mathrm{e}^{-B} \pm \nabla(4 B+\phi)\right)^{2}= & {\left[\gamma^{-2} M^{2}+\beta^{-2}(\nabla \alpha)^{2}\right] } \\
(\nabla(3 \phi-4 C-4 D))^{2}= & \left(4 e^{-D-C}-(p-2) \beta^{-1} \nabla \alpha\right)^{2} \\
& +(p-2)^{2} \gamma^{-2} M^{2},
\end{aligned}
$$

where

$$
\begin{aligned}
& \beta=\mathrm{e}^{(p+1) A+\frac{p-3}{4} \phi}, \\
& \gamma=\mathrm{e}^{(6-p) B-\frac{p-1}{4} \phi} .
\end{aligned}
$$

In addition to these equations we must supplement also the Bianchi identity for $F_{8-p}$ in equation (4.6). We have verified that this system reproduces the one presented in [1] for $p=6$ for which no $B$ warp factor is present. For $p=5$ the system is also modified as the third equation takes the form

$$
(\nabla(4 B+\phi))^{2}=\left[\gamma^{-2} M^{2}+\beta^{-2}(\nabla \alpha)^{2}\right] .
$$

This is due to the fact that for general $p$ the metric has a factor $S^{6-p}$ which for $p=5$ is simply a circle and the associated Ricci tensor must vanish. 
The first two BPS equations can be combined so that $\nabla \alpha$ does not appear,

$$
(p-3)^{2}(\nabla(4 A+\phi))^{2}-16(\nabla \phi)^{2}=-16(p-3)^{2} \mathrm{e}^{-2 A}+4 \gamma^{-2} M^{2}\left(\lambda^{2}-p+2\right) .
$$

From the equations of motion one can deduce:

$$
-4 p(p-3) \mathrm{e}^{-2 A}-4(p-3) \nabla^{2} A+(7-p) \nabla^{2} \phi=-2 \gamma^{-2}\left(\beta^{-2} \alpha^{2}-1\right) .
$$

Expanding these two equations around a $\mathrm{D} p$ singularity, we obtain an expression for the constant $k_{0}$ which was introduced in section 4.3:

$$
\frac{1}{2} k_{0}^{2}=\frac{(p-4)(p-3)^{2}}{(p-5)} .
$$

This equation has important consequences because for $p=3$ and $p=4$ we see that $\alpha_{0}$ vanishes (cf. equation (4.25)). The fact that $\alpha_{0}$ vanishes is however in direct contradiction with our previous result that $\alpha_{0}$ is proportional to the non-zero CC. We must conclude that the assumption made when deriving the relation between $\alpha_{0}$ and the CC; that the internal space is compact, is not true for $p=3,4$. This result only holds when we assume a $\mathrm{D} p$ singularity at $\rho=0$, a compact solution with no brane at the pole might of course exist.

Open Access. This article is distributed under the terms of the Creative Commons Attribution License (CC-BY 4.0), which permits any use, distribution and reproduction in any medium, provided the original author(s) and source are credited.

\section{References}

[1] F. Apruzzi, M. Fazzi, D. Rosa and A. Tomasiello, All AdS $S_{7}$ solutions of type-II supergravity, JHEP 04 (2014) 064 [arXiv: 1309.2949] [INSPIRE].

[2] D. Junghans, D. Schmidt and M. Zagermann, Curvature-induced Resolution of Anti-brane Singularities, JHEP 10 (2014) 34 [arXiv:1402.6040] [INSPIRE].

[3] P. McGuirk, G. Shiu and Y. Sumitomo, Non-supersymmetric infrared perturbations to the warped deformed conifold, Nucl. Phys. B 842 (2011) 383 [arXiv:0910.4581] [INSPIRE].

[4] I. Bena, M. Graña and N. Halmagyi, On the Existence of Meta-stable Vacua in Klebanov-Strassler, JHEP 09 (2010) 087 [arXiv: 0912.3519] [INSPIRE].

[5] I. Bena, G. Giecold, M. Graña, N. Halmagyi and S. Massai, The backreaction of anti-D3 branes on the Klebanov-Strassler geometry, JHEP 06 (2013) 060 [arXiv:1106.6165] [INSPIRE].

[6] S. Kachru, J. Pearson and H.L. Verlinde, Brane/flux annihilation and the string dual of a nonsupersymmetric field theory, JHEP 06 (2002) 021 [hep-th/0112197] [INSPIRE].

[7] S. Kachru, R. Kallosh, A.D. Linde and S.P. Trivedi, De Sitter vacua in string theory, Phys. Rev. D 68 (2003) 046005 [hep-th/0301240] [INSPIRE].

[8] S. Kachru, R. Kallosh, A.D. Linde, J.M. Maldacena, L.P. McAllister and S.P. Trivedi, Towards inflation in string theory, JCAP 10 (2003) 013 [hep-th/0308055] [INSPIRE]. 
[9] J. Blåbäck, U.H. Danielsson, D. Junghans, T. Van Riet, T. Wrase and M. Zagermann, The problematic backreaction of SUSY-breaking branes, JHEP 08 (2011) 105 [arXiv:1105.4879] [INSPIRE].

[10] J. Blåbäck, U.H. Danielsson, D. Junghans, T. Van Riet, T. Wrase and M. Zagermann, (Anti-)Brane backreaction beyond perturbation theory, JHEP 02 (2012) 025 [arXiv:1111.2605] [INSPIRE].

[11] D. Gaiotto and A. Tomasiello, Holography for (1,0) theories in six dimensions, JHEP 12 (2014) 003 [arXiv: 1404.0711] [INSPIRE].

[12] O. DeWolfe, S. Kachru and H.L. Verlinde, The Giant inflaton, JHEP 05 (2004) 017 [hep-th/0403123] [INSPIRE].

[13] J. Blåbäck, U.H. Danielsson and T. Van Riet, Resolving anti-brane singularities through time-dependence, JHEP 02 (2013) 061 [arXiv:1202.1132] [INSPIRE].

[14] B. Michel, E. Mintun, J. Polchinski, A. Puhm and P. Saad, Remarks on brane and antibrane dynamics, arXiv:1412.5702 [INSPIRE].

[15] F.F. Gautason, D. Junghans and M. Zagermann, Cosmological Constant, Near Brane Behavior and Singularities, JHEP 09 (2013) 123 [arXiv: 1301.5647] [INSPIRE].

[16] J. Blåbäck, U.H. Danielsson, D. Junghans, T. Van Riet and S.C. Vargas, Localised anti-branes in non-compact throats at zero and finite T, JHEP 02 (2015) 018 [arXiv:1409.0534] [INSPIRE].

[17] J. Blåbäck, U.H. Danielsson, D. Junghans, T. Van Riet, T. Wrase and M. Zagermann, Smeared versus localised sources in flux compactifications, JHEP 12 (2010) 043 [arXiv: 1009.1877] [INSPIRE].

[18] S.B. Giddings, S. Kachru and J. Polchinski, Hierarchies from fluxes in string compactifications, Phys. Rev. D 66 (2002) 106006 [hep-th/0105097] [INSPIRE].

[19] U.H. Danielsson and T. Van Riet, Fatal attraction: more on decaying anti-branes, JHEP 03 (2015) 087 [arXiv: 1410.8476] [INSPIRE].

[20] I. Bena, A. Buchel and O.J.C. Dias, Horizons cannot save the Landscape, Phys. Rev. D 87 (2013) 063012 [arXiv:1212.5162] [INSPIRE].

[21] I. Bena, J. Blåbäck, U.H. Danielsson and T. Van Riet, Antibranes cannot become black, Phys. Rev. D 87 (2013) 104023 [arXiv:1301.7071] [INSPIRE].

[22] G.S. Hartnett, Localised anti-branes in flux backgrounds, JHEP 06 (2015) 007 [arXiv: 1501.06568] [INSPIRE].

[23] J. Polchinski and M.J. Strassler, The String dual of a confining four-dimensional gauge theory, hep-th/0003136 [INSPIRE].

[24] I. Bena, D. Junghans, S. Kuperstein, T. Van Riet, T. Wrase and M. Zagermann, Persistent anti-brane singularities, JHEP 10 (2012) 078 [arXiv:1205.1798] [INSPIRE].

[25] I. Bena, M. Graña, S. Kuperstein and S. Massai, Polchinski-Strassler does not uplift Klebanov-Strassler, JHEP 09 (2013) 142 [arXiv:1212.4828] [INSPIRE].

[26] E. Silverstein, TASI/PiTP/ISS lectures on moduli and microphysics, hep-th/0405068 [INSPIRE].

[27] E. D'Hoker, J. Estes and M. Gutperle, Exact half-BPS Type IIB interface solutions. I. Local solution and supersymmetric Janus, JHEP 06 (2007) 021 [arXiv:0705.0022] [INSPIRE]. 
[28] J.M. Maldacena and H.S. Nastase, The Supergravity dual of a theory with dynamical supersymmetry breaking, JHEP 09 (2001) 024 [hep-th/0105049] [INSPIRE].

[29] I.R. Klebanov and M.J. Strassler, Supergravity and a confining gauge theory: duality cascades and chi SB resolution of naked singularities, JHEP 08 (2000) 052 [hep-th/0007191] [INSPIRE].

[30] I. Bena, G. Giecold, M. Graña, N. Halmagyi and S. Massai, On metastable vacua and the warped deformed conifold: analytic results, Class. Quant. Grav. 30 (2013) 015003 [arXiv: 1102.2403] [INSPIRE].

[31] R.C. Myers, Dielectric branes, JHEP 12 (1999) 022 [hep-th/9910053] [InSPIRE].

[32] O. Aharony, Y.E. Antebi and M. Berkooz, Open string moduli in KKLT compactifications, Phys. Rev. D 72 (2005) 106009 [hep-th/0508080] [InSPIRE].

[33] I. Bena, M. Graña, S. Kuperstein and S. Massai, Giant Tachyons in the Landscape, JHEP 02 (2015) 146 [arXiv: 1410.7776] [INSPIRE].

[34] U.H. Danielsson, Perturbative decay of anti-branes in flux backgrounds due to space time instabilities, arXiv:1502.01234 [INSPIRE].

[35] I. Bena, A. Puhm and B. Vercnocke, Metastable supertubes and non-extremal black hole microstates, JHEP 04 (2012) 100 [arXiv: 1109.5180] [INSPIRE].

[36] I. Bena, A. Puhm and B. Vercnocke, Non-extremal black hole microstates: fuzzballs of fire or fuzzballs of fuzz?, JHEP 12 (2012) 014 [arXiv:1208.3468] [INSPIRE].

[37] E. Bergshoeff, R. Kallosh, T. Ortín, D. Roest and A. Van Proeyen, New formulations of $D=10$ supersymmetry and D8-O8 domain walls, Class. Quant. Grav. 18 (2001) 3359 [hep-th/0103233] [INSPIRE]. 